\title{
Adaptive Backstepping Method for Stabilizing Systems of Fractional Order Ordinary Differential Equations
}

\author{
Asad J. Taher ${ }^{1, *}$, Fadhel S. Fadhel ${ }^{2}$ and Nabaa N. Hasan ${ }^{1}$ \\ ${ }^{1}$ Department of Mathematics, College of Science, Mustansiriyah University, Baghdad-Iraq \\ ${ }^{2}$ Department of Mathematics and Computer Applications, College of Science, Al-Nahrain University, \\ Baghdad-Iraq
}

\begin{tabular}{|c|c|}
\hline Article's Information & Abstract \\
\hline $\begin{array}{l}\text { Received: } \\
\text { 03-10-2021 } \\
\text { Accepted: } \\
\text { 14-11-2021 } \\
\text { Published: } \\
\text { 31-12-2021 }\end{array}$ & $\begin{array}{l}\text { In this paper the method of adaptive backstepping for stabilizing and solving } \\
\text { system of ordinary and partial differential equations will be used and applied to } \\
\text { investigate and study the stability linear systems of Caputo fractional order } \\
\text { ordinary differential equations. The basic idea of this approach is to find a } \\
\text { quadratic Lyapunov functions for stabilizing the subsystems. }\end{array}$ \\
\hline $\begin{array}{l}\text { Keywords: } \\
\text { Backstepping method } \\
\text { Fractional differential equations } \\
\text { Stability } \\
\text { Lyapunov method }\end{array}$ & \\
\hline $\begin{array}{l}\text { DOI: } 10.22401 / \text { ANJS.24.4.07 } \\
{ }^{*} \text { Corresponding author: asadjassi }\end{array}$ & 76@@gmail.com \\
\hline
\end{tabular}

\section{Introduction}

Fractional calculus began around 300 years ago, on September 30, 1695, when Gottfried Leibniz received a letter from G. A. L'Hôpital, which answered the question "what meaning of the derivative of order $1 / 2$ ?" However, fractional calculus did not develop in the same way as integer calculus until the twentieth century, when life became more complex and a slew of scientific inventions improved scientific research tools. As a result, scientists developed a slew of fractional calculus methods, which are now used in fields such as engineering and communications, etc. plays a key role in many sectors of research, particularly engineering, where the study of fractional differential equations stability theory is essential [10].

Branches of fractional calculus in different fields were started earlier in 1695 , but the classical calculus found numerous applications in science and engineering, which affect later on fractional calculus to have so many applications which are solved successfully that are difficult to be solved using classical calculus. Fractional calculus remained inactive until the twentieth century, when it became necessary to simulate real-world issues in fields like as biology, bioengineering, astronomy, physics, and engineering $[1,2]$. Hence after, fractional calculus, followed by fractional order ordinary differential equations (FODEs), is a crucial subject in applied mathematics [3-5]. In continuative to what was mentioned above, stability analysis FODEs is more difficult to study than the stability analysis in ordinary differential equations (ODEs), because fractional derivatives have weakly singular kernels and nonlocal [4-6]. Also, as is well known the dynamical system is stable when it is allowed to perform persistent tiny oscillations about the state of motion, or around the system equilibrium. This notion may be utilized in a variety of ways to examine the stability of ODEs in general and FODEs in particular. The first approach uses eigenvalues, whereas the second was entrenched by Lyapunov at the end of the 19th century and was successfully applied to whole whose stability can be studied using the first approach, and even so for those problems in which the first approach failed to be applied problems. The direct method of Lyapunov's is abbreviated as the direct method, since it may be used, directly to differential conditions with solution's no prior knowledge and applied [8]. Beyond this method, the primary concept is to develop a scalar function, say $\mathrm{V}$, that meets certain stated requirements in order to assess the stability of the system of ODEs [6].

Matignon was the first researcher how in present stability results linked to the restricted modeling of FODEs in his Ph.D. thesis in 1994 [5,8]. There are also a number of significant conclusions relating to the linear system of FODEs with Caputo fractional derivative of order $\alpha$, where $0<\alpha \leq 1$, such as, Qian et al. in 2012 [8] examined the linear FODEs were he used Riemann-Liouville fractional order derivative. Following that, a number of scholars looked at the stability of nonlinear FODEs with fractional order derivatives between 0 and 1 , see for more information [6]. 


\section{Al-Nahrain Journal of Science}

ANJS, Vol.24 (4), December, 2021, pp. 46-51

It is also worth noting that, owing to the complexity of the ODEs or FODEs, using trajectories to verify the asymptotic stability of the system is not always viable. Backstepping is a nonlinear control technique for stabilizing systems of differential equations, whether ordinary or partial, in which it is based on the concept of introducing a set of intermediate variables and the procedure of precisely given negativity of Lyapunov function derivatives that help us to build a common control Lyapunov function for the system [7-9]. Because of this nature, the backstepping technique is easy applied method to different classes of systems, where there are different types of backstepping method depending on the number of control functions introduced in the system. The first type of backstepping has virtual variables and control function in the last equation of dynamic system, which is called the adaptive backstepping method while the second type it has virtual variables and control functions in each equation $n$ of the dynamic system[7,9-11].

In this work, we aim to determine the Lyapunov characterization function for the asymptotic stability of the solution of the FODEs defined by the adaptive backstepping approach in this study we will explore. The backstepping stabilization approach that has been refined and enhanced to be more relevant for systems Caputo FODE's in order to obtain asymptotically stable solutions.

\section{Preliminaries}

The concepts of stability, control and solution of specific systems are of excellent importance in many real-life problems. Therefore, their basic essential notions appeared to be required to comprehend such issues; and thus, we will provide some of them in this part, which are required for the remainder of this article.

Obviously, we start first with the basic fundamental definitions in fractional calculus of integration and derivatives of those which will be used in this work.

Definition 1, [12-15]. The left and right Riemann-Liouville fractional integrals of order $\alpha \in R^{+}, n-1<\alpha \leq n, n \in N$ of continuous function $y$ over certain domain are given respectively by:

$$
{ }^{R L} I_{a, t}^{\alpha} y(t)=\frac{1}{\Gamma(\alpha)} \int_{a}^{t}(t-\tau)^{\alpha-1} y(\tau) d \tau
$$

and

$$
{ }^{R L} I_{a, t}^{\alpha} y(t)=\frac{1}{\Gamma(\alpha)} \int_{t}^{b}(\tau-t)^{\alpha-1} y(\tau) d \tau
$$

where $\Gamma$ stands for the standard gamma function.

Definition 2, [16,17]. The left and right Riemann-Liouville fractional derivative of order $\alpha \in \mathbb{R}^{+}, n-1<\alpha \leq n, n \in$ $N$ of continuous function $y$ over certain domain are defined respectively as:

$$
\begin{aligned}
& { }^{R L} D_{a, t}^{\alpha} y(t)=\frac{1}{\Gamma(n-\alpha)} \frac{d^{n}}{d x^{n}} \int_{a}^{t}(t-\tau)^{\alpha-1} y(\tau) d \tau \\
& \text { and } \\
& \qquad{ }^{R L} D_{a, t}^{\alpha} y(t)=\frac{(-1)^{n}}{\Gamma(n-\alpha)} \frac{d^{n}}{d x^{n}} \int_{t}^{b}(\tau-t)^{\alpha-1} y(\tau) d \tau
\end{aligned}
$$

Definition 3, [12, 18]. The left and right Caputo fractional derivative of order $\alpha \in \mathbb{R}^{+}, n-1<\alpha \leq n, n \in N$ of a continuous function $y$ over a certain domain are defined respectively by:

$$
{ }_{a}^{C} D_{t}^{\alpha} y(x)=\frac{1}{\Gamma(n-\alpha)} \int_{a}^{x} \frac{1}{(x-\tau)^{\alpha-n}} y^{(n)}(t) d \tau
$$

and

$$
{ }_{t}^{C} D_{b}^{\alpha} y(t)=\frac{(-1)^{\alpha}}{\Gamma(n-\alpha)} \int_{x}^{b} \frac{1}{(\tau-t)^{\alpha-n}} y^{(n)}(\tau) d \tau
$$

The considered system of FODEs is of Caputo fractional derivative, which has the form:

$$
{ }_{t_{0}}^{C} D_{x}^{\alpha} x(t)=f(t, x(t)), x\left(t_{0}\right)=x_{0}
$$

where $\alpha \in(0,1], f:\left[t_{0}, \infty\right] \times \Omega \rightarrow R^{n}$ is a piecewise continuous function in t, locally Lipschitzian in $\mathrm{x}$ and $\Omega \subseteq$ $\mathbb{R}^{n}$ is a certain domain that contains the origin. Also, it is necessary to recall that $x^{*}$ is an equilibrium point of the dynamic system Error! Reference source not found. if $f\left(t, x^{*}\right)=0$.

The direct methods of stability may be used to study quantitively behavior of system Error! Reference source not found. constructing the Lyapunov function, which must ensure the modified system's asymptotic stability and, is one of the essential instruments in the backstepping approach. Lyapunov functions are scalar functions used to show and establish the stability of solutions or a system's equilibrium point, assuming they exist and are not unique. Before entering into and introducing the major topic of this article, we need a more additional concepts to refresh our minds on the Lyapunov functions.

If a continuous function $V(t)>0$ for all $t \neq 0$, then $V$ is called positive definite and where a function $V(t)<$ 0 , for all $t \neq 0$, is called negative definite. The main theorem of stability using Lyapunov direct method is that stated next:

Theorem 1, [27-30]. Suppose that $V$ is a scalar continuous real valued function of the state variables $t_{1}, t_{2}, \ldots, t_{n}$ defined on the region $\Omega$ containing the origin and if:

i. $V(t)>0, \forall t \in \Omega$ and $\dot{V}(t) \leq 0$, then the zero solution is stable.

ii. $V(t)>0, \forall t \in \Omega$ and $\dot{V}(t)<0, \forall t \in \Omega$, then the zero solution is asymptotically stable.

iii. $V(t)>0, \forall t \in \Omega$ and $\dot{V}(t)>0, \forall t \in \Omega$, then the zero solution is unstable.

Regarding Lyapunov functions, we have the following conclusions for the fractional order derivative.

Theorem 2, [7]. Suppose a Lyapunov function defined as $V=\frac{1}{2} z^{2}$, where the variable of interest is z. If $z_{0}^{C} D_{t}^{\alpha} z<$ 0 , for all $0<\alpha \leq 1$ is ensured, then $z \dot{Z}<0$ is satisfied.

In the next theorem, the relation between Lyapunov function and Caputo fractional order derivative will be given. 


\section{Al-Nahrain Journal of Science}

ANJS, Vol.24 (4), December, 2021, pp. 46-51

Theorem 3, [17]. Suppose Lyapunov function $V(t) \in R$, which has Caputo fractional derivative of order $\alpha, 0<\alpha \leq$ 1 , then:

$$
\frac{1}{2}{ }_{0}^{C} D_{t}^{\alpha} V^{2}(t) \leq V(t){ }_{0}^{C} D_{t}^{\alpha} V(t), \forall t>0
$$

Theorem 4, [17]. If $t=0$ is an equilibrium point of the Caputo fractional order autonomous system:

$$
{ }_{0}^{C} D_{t}^{\alpha} y(t)=f(y(t)), 0<\alpha \leq 1
$$

where $y(t) \in R$. Then:

a) If $y(t){ }_{0}^{C} D_{t}^{\beta} y(t) \leq 0$, then the point $y=0$ is stable.

b) If $y(t){ }_{0}^{C} D_{t}^{\beta} y(t)<0$, then the point $y=0$ is asymptotically stable.

\section{The Adaptive Backstepping Method for System of FODE's}

For stabilizability and solvability of a specific FODEs system of the form:

$$
\left.\begin{array}{c}
{ }_{0}^{C} D_{t}^{\alpha_{1}} y_{1}=f_{1}\left(y_{1}, y_{2}\right) \\
{ }_{0}^{{ }_{0}} D_{t}^{a_{2}} y_{2}=f_{2}\left(y_{1}, y_{2}, y_{3}\right) \\
\vdots \\
{ }_{0}^{c} D_{t}^{a_{n}} y_{n}=f_{n}\left(y_{1}, y_{2}, \ldots, y_{n}\right)
\end{array}\right\}
$$

where $0<\alpha_{i} \leq 1, i=1,2, \ldots, \mathrm{n}$. We add a controller function $u$ to be applied for the last equation of the FODEs the adaptive backstepping method to a specific system as a result, this system of fractional order derivatives will take the following structure:

$$
\left.\begin{array}{c}
{ }_{0}^{c} D_{t}^{\alpha_{1}} y_{1}=f_{1}\left(y_{1}, y_{2}\right) \\
{ }_{0}^{c} D_{t}^{a_{2}} y_{2}=f_{2}\left(y_{1}, y_{2}, y_{3}\right) \\
\vdots \\
{ }_{0}^{c} D_{t}^{a_{n}} y_{n}=f_{n}\left(y_{1}, y_{2}, \ldots, y_{n}\right)+u(t)
\end{array}\right\}
$$

where $y(t)=\left(y_{1}(t), y_{2}(t), \ldots, y_{n}(t)\right) \epsilon R^{n}$ is the state vector of the system, $\alpha_{i} \in(0,1], f_{i}, i=1,2, \ldots, n$ are continuous functions and $u$ is the controller input functions, which will be introduced for the purpose for asymptotically stabilizing the original system of FODEs asymptotically and as a consequence find its solution [28-30].

Now the objective is to apply the backstepping method in order to design a state feedback control function, which asymptotically stabilizes the origin system. The design procedure may for simplify approach divided into $\mathrm{n}-$ steps, as it is given in the next theorem:

Theorem 5. Suppose the system of FODEs Error! Reference source not found., with state variable $x \in \mathbb{R}^{n}$ and controller function $u: \mathbb{R}^{n} \rightarrow \mathbb{R}$. If the Lyapunov functions of the lower subsystems of the FODEs system Error! Reference source not found. are supposed to be:

$$
\left.\begin{array}{l}
V_{1}\left(z_{1}\right)=p_{1} z_{1} p_{1}, p_{1} \in \mathbb{R}^{+}, z_{1}(t) \in \mathbb{R} \\
V_{i}\left(z_{1}, z_{2}, \ldots, z_{i}\right)=V_{i-1}\left(z_{1}, z_{2}, \ldots, z_{i-1}\right)+p_{i} z_{i} p_{i}
\end{array}\right\}
$$

Where $p_{i} \in \mathbb{R}^{+}, z_{i}(t) \in \mathbb{R}, i=2,3, \ldots, n$, then there exists a nonlinear controller function $u$, which make system Error! Reference source not found. asymptotically stable and solvable, where:

$$
\beta_{i}\left(z_{1}, z_{2}, \ldots, z_{i}\right)=f_{i}\left(z_{1}, z_{2}, \ldots, z_{i}, x_{i+1}, \ldots, x_{n}\right)-z_{i}
$$

for all $i=2,3, \ldots, n$.

Proof. As it is followed in the backstepping method, for simplicity and comparison purpose the proof will be breakdown into steps as follows:

Step 1: We will discuss the stability of first subsystem by taking the first equation of system of Error! Reference source not found., namely:

$$
{ }_{0}^{C} D_{t}^{\beta_{1}} y_{1}=f_{1}\left(y_{1}, y_{2}\right)
$$

where $y_{2}$ represents a virtual controller introduced for stability, and define $y_{1}=z_{1}$, then derive this transformation both sides, with fractional order $\beta_{1}$ using Caputo fractional order derivative with respect to time $t$, to get:

$$
{ }_{0}^{C} D_{t}^{\beta_{1}} z_{1}={ }_{0}^{C} D_{t}^{\beta_{1}} y_{1}=f_{1}\left(z_{1}, y_{2}, \ldots, y_{n}\right)
$$

assuming the first Lyapunov function in quadratic form as:

$$
V_{1}\left(z_{1}\right)=\frac{1}{2} z_{1}^{2}=z_{1}{ }^{T} p_{1} z_{1}, p_{1} \in \mathbb{R}^{+}
$$

with its derivative, with respect of time $t$ is:

$$
\dot{V}_{1}\left(z_{1}\right)=z_{1} \dot{z}_{1}=-z_{1}^{T} q_{1} z_{1}<0
$$

where $q_{1}$ is a positive constant. Since it is required that $z_{1}{ }_{0}^{C} D_{t}^{\alpha_{1}} z_{1}<0$ that define by using Theorem $2, z_{1} \dot{z_{1}}<$ 0 that is given by using Theorem 3 and $4, \dot{V}_{1}\left(z_{1}\right)$ be a negative definite function in $\mathbb{R}^{n}$, with application of Lyapunov stability theory, then equation Error! Reference source not found. will be asymptotically stable. It is clear that if we take the virtual control $y_{2}=\beta_{1}\left(z_{1}\right)$, then equation Error! Reference source not found. is asymptotically stable. The function $\beta_{1}\left(z_{1}\right)$ had better to be assessed while $z_{2}=0$ is regarded as a controller.

Step 2: To make the second equation of system Error! Reference source not found. stable, define the error function between $y_{2}$ and $\beta_{1}\left(z_{1}\right)$ to be as follows:

$$
z_{2}=y_{2}-\beta_{1}\left(z_{1}\right)
$$

hence the time derivative of the error dynamics of subsystem Error! Reference source not found., is given by:

$$
\left.\begin{array}{l}
{ }_{0}^{C} D_{t}^{\alpha_{1}} z_{1}=f_{1}\left(z_{1}, y_{2}, y_{3} \ldots, y_{n}\right) \\
{ }_{0}^{C} D_{t}^{\alpha_{2}} z_{2}=f_{1}\left(z_{1}, z_{2}+\beta_{1}\left(z_{1}\right), y_{3} \ldots, y_{n}\right)-{ }_{0}^{C} D_{t}^{\alpha_{2}} \beta\left(z_{1}\right)
\end{array}\right\}
$$

where $x_{3}$ is a virtual controller of subsystem Error! Reference source not found., which is chosen so that to stabilize this subsystem and suppose it is equal to $\beta_{1}\left(z_{1}\right)$.

In order to find the second Lyapunov function $V_{2}$, which stabilizes asymptotically equation Error! Reference source not found., suppose $V_{2}$ to be defined by:

$$
\begin{aligned}
V_{2}\left(z_{1}, z_{2}\right) & =\frac{1}{2} z_{1}^{2}+\frac{1}{2} z_{2}^{2} \\
& =V_{1}+z_{2}^{T} p_{2} z_{2}, p_{2} \in \mathbb{R}^{+}
\end{aligned}
$$

then the derivative of $V_{2}$ with respect to time $t$ is:

$$
\begin{aligned}
\dot{V}_{2}\left(z_{1}, z_{2}\right) & =z_{1} \dot{z}_{1}+z_{2} \dot{z}_{2} \\
& =-z_{1}{ }^{T} q_{1} z_{1}-z_{2}{ }^{T} q_{2} z_{2}<0
\end{aligned}
$$

where $q_{1}$ and $q_{2}$ are positive constants. By chosen suitable value of $\beta_{1}\left(z_{1}\right)$ to make $z_{2}{ }_{0}^{C} D_{t}^{\beta_{2}} z_{2}<0$, we get:

$$
z_{1}{ }_{0}^{C} D_{t}^{\beta_{1}} z_{1}+z_{2}{ }_{0}^{C} D_{t}^{\beta_{2}} z_{2}<0
$$

Since that by using Theorem 2 , it is known that:

$$
z_{1} \dot{z}_{1}+z_{2} \dot{z_{2}}<0
$$




\section{Al-Nahrain Journal of Science}

ANJS, Vol.24 (4), December, 2021, pp. 46-51

we get $\dot{V}_{2}\left(z_{1}, z_{2}\right)$ is a negative definite function in $\mathbb{R}^{n}$ and by using Lyapunov stability theory with Theorems 3 and 4, then the subsystem Error! Reference source not found. is asymptotically stable. Similarly, if the virtual control is taken to be $y_{3}=\beta_{2}\left(z_{1}, z_{2}\right)$ is assumed to the subsystem Error! Reference source not found. asymptotically stable. Step n: As in the above steps, the same approach will be followed, we arrive at the $\mathrm{n}^{\text {th }}$ step by defining the error function $z_{n}$ as:

$$
z_{n}=y_{n}-\beta_{n-1}\left(z_{1}, z_{2}, \ldots, z_{n}\right)
$$

and suppose that $\mathrm{z}_{1}, \mathrm{z}_{2}, \ldots, \mathrm{z}_{\mathrm{n}}$ are the state variables of the overall transformed system is given by:

$$
\begin{aligned}
{ }_{0}^{C} D_{t}^{\beta_{1}} z_{1}= & f_{1}\left(z_{1}, x_{2}, y_{3} \ldots, y_{n}\right) \\
{ }_{0}^{C} D_{t}^{\alpha_{2}} z_{2}= & f_{1}\left(z_{1}, z_{2}+\beta_{1}\left(z_{1}\right), y_{3} \ldots, y_{n}\right)-{ }_{0}^{C} D_{t}^{\alpha_{2}} \beta_{1}\left(z_{1}\right) \\
\vdots & \\
{ }_{0}^{C} D_{t}^{\alpha_{n}} z_{n}= & f_{n}\left(z_{1}, z_{2}+\beta_{1}\left(z_{1}\right), \ldots, z_{n}++\beta_{n-1}\left(z_{1}, z_{2}, \ldots,\right.\right. \\
& \left.\left.z_{n-1}\right)\right)-{ }_{0}^{C} D_{t}^{\beta_{n}} \beta_{n-1}\left(z_{1}, z_{2}, \ldots, z_{n}\right)+u
\end{aligned}
$$

and define Lyapunov function as:

$$
\begin{aligned}
V_{n}\left(z_{1}, z_{2}, \ldots, z_{n}\right)= & \frac{1}{2} z_{1}^{2}+\frac{1}{2} z_{2} z_{2}^{2}+\cdots+\frac{1}{2} z_{n-1}^{2}+\frac{1}{2} z_{n}^{2} \\
= & V_{n-1}\left(z_{1}, z_{2}, \ldots, z_{n-1}\right)+ \\
& z_{n}^{T} p_{n} z_{n}, p_{n} \in \mathbb{R}^{+}
\end{aligned}
$$

Also, differentiate $V_{n}$ with respect to time $t$, give:

$$
\begin{aligned}
\dot{V}_{n}\left(z_{1}, z_{2}, \ldots, z_{n}\right) & =z_{1} \dot{z}_{1}+z_{2} \dot{z_{2}}+\cdots+z_{n} \dot{z_{n}} \\
& =-z_{1}{ }^{T} q_{1} z_{1}-z_{2}{ }^{T} q_{2} z_{2}-\cdots-z_{n}{ }^{T}
\end{aligned}
$$

where $q_{1}, q_{2}, q_{3}, \ldots, q_{n}$ are positive constants. Also, here we will get $z_{1} \dot{z_{1}}+z_{2} \dot{z_{2}}+\cdots+z_{n} \dot{z_{n}}<0$, when we choose suitable controller function $u$ and:

$$
z_{1}{ }_{0}^{C} D_{t}^{\alpha_{1}} z_{1}+z_{2}{ }_{0}^{C} D_{t}^{\alpha_{2}} z_{2}+\cdots+z_{n}{ }_{0}^{C} D_{t}^{\alpha_{n}} z_{n}<0
$$

then by using Theorem $2, z_{1} \dot{z}_{1}+z_{2} \dot{z_{2}}+\cdots+z_{n} \dot{z_{n}}<0$. and hence $\dot{V}_{n}$ is by using Theorem 3 and 4 a negative definite function in $R^{n}$ and similarly by Lyapunov stability theory, subsystem Error! Reference source not found. is asymptotically stable. The virtual control:

$$
y_{n}=\alpha_{n-1}\left(z_{1}, z_{2}, \ldots, z_{n-1}\right)
$$

may be evaluated, which makes subsystem Error! Reference source not found. is asymptotically stable. Thus, as a result of the above steps, system Error! Reference source not found. is globally asymptotically stable for all initial condition $x_{i}(0) \in \mathbb{R}^{n}, i=1,2, \ldots, n$.

\section{Applications}

In this section, two examples are considered as an application to illustrate the validity and applicability of the proposed approach for stabilizing systems of Caputo fractional order.

Example 1. Consider the homogeneuous linear system of Caputo FODEs:

$$
\left.\begin{array}{l}
{ }_{0}^{C} D_{t}^{\alpha_{1}} y_{1}(t)=2 y_{2} \\
{ }_{0}^{C} D_{t}^{\alpha_{2}} y_{2}(t)=-y_{1}-3 y_{2}
\end{array}\right\}
$$

by using backstepping method and in order to asymptotically stabilize this system, even so it is stable or not, introduce the controller functions $\beta_{1}$ and $\beta_{2}$, as follows:

$$
\left.\begin{array}{l}
{ }_{0}^{C} D_{t}^{\alpha_{1}} y_{1}(t)=2 y_{2} \\
{ }_{0}^{C} D_{t}^{\alpha_{2}} y_{2}(t)=-y_{1}-3 y_{2}+u
\end{array}\right\}
$$

Step 1: Suppose $z_{1}=y_{1}$, then the Caputo derivative of order $\alpha_{1}$ with respect to $t$ for the first equation of system Error! Reference source not found. will be:

$$
{ }_{0}^{C} D_{t}^{\beta_{1}} z_{1}(t)=2 y_{2}
$$

and when we suppose that $z_{2}=y_{2}-\beta_{1}$, then equation Error! Reference source not found. will be:

$$
{ }_{0}^{C} D_{t}^{\alpha_{1}} z_{1}(t)=2 z_{2}+2 \beta_{1}
$$

to prove that $z_{1}{ }_{0}^{C} D_{t}^{\alpha} z_{1}$ is negative definite, where the controller function $\beta_{1}$ is chosen as $\beta_{1}=-2 z_{2}-k_{1} z_{1}$. If we substitute in Error! Reference source not found. then equation Error! Reference source not found. will be:

$$
z_{1}{ }_{0}^{C} D_{t}^{\alpha_{1}} z_{1}=-2 k_{1} z_{1}^{2}
$$

which is negative everywhere when $k_{1}$ is a positive constant. Since $z_{1}{ }_{0}^{C} D_{t}^{\beta_{1}} z_{1}<0$, the by using Theorem 2 implies $z_{1} \dot{z}_{1}<0$, then choosing the Lyapunov function $V_{1}=\frac{1}{2} z_{1}^{2}$ with $\dot{V}_{1}=z_{1} \dot{z}_{1}$ that means equation (30) will be asymptotically stable. The function $\beta_{1}$ should be estimated while $z_{2}=0$ is considered as a controller.

Step 2: Suppose $z_{2}=y_{2}-\beta_{1}\left(z_{1}\right)$ or equivalently by $y_{2}=$ $z_{2}+\beta_{1}\left(z_{1}\right)$, then the Caputo derivative of both sides of $z_{2}$ with order $\beta_{2}$ will be:

$$
{ }_{0}^{C} D_{t}^{\alpha_{2}} y_{2}={ }_{0}^{C} D_{t}^{\alpha_{2}} z_{2}+{ }_{0}^{C} D_{t}^{\alpha_{2}} \beta_{1}
$$

then the second differential equation of the system Error!

Reference source not found. will be:

$$
{ }_{0}^{C} D_{t}^{\alpha_{2}} z_{2}(t)=-z_{1}-3 z_{2}-3 \beta_{1}+{ }_{0}^{C} D_{t}^{\alpha_{2}} \beta_{1}+u
$$

and thus:

$$
z_{2}{ }_{0}^{C} D_{t}^{\alpha_{2}} z_{2}=z_{2}\left(z_{1}-3 z_{2}-3 \beta_{1}\left(z_{1}\right)+{ }_{0}^{C} D_{t}^{\alpha_{2}} \beta_{1}+u\right)
$$

to make $z_{2}{ }_{0}^{C} D_{t}^{\alpha_{2}} z_{2}$ negative definite, choose:

$$
u=-z_{1}+3 z_{1}+3 \beta_{1}\left(z_{1}\right)-{ }_{0}^{C} D_{t}^{\alpha_{2}} \beta_{1}-k_{2} z_{2}
$$

then the equation will be as:

$$
\begin{gathered}
{ }_{0}^{C} D_{t}^{\alpha_{2}} z_{2}=-k_{1} z_{2} \\
\text { and } \\
{ }_{2}{ }_{2}{ }_{0}^{C} D_{t}^{\alpha_{2}} z_{2}=-k_{2} z_{2}^{2}
\end{gathered}
$$

which is negative everywhere at $k_{2}$ positive constant. By using Theorem 2 implies to $z_{2} \dot{z}_{2}<0$, that will give:

$$
z_{1} \dot{z}_{1}+z_{2} \dot{z}_{2}<0 \text { and } z_{1}{ }_{0}^{C} D_{t}^{\alpha_{1}} z_{1}+z_{2}{ }_{0}^{C} D_{t}^{\alpha_{2}} z_{2}<0
$$

then we can choose a Lyapunov function $\mathrm{V}$ as:

such that:

$$
\begin{aligned}
V_{2}(t) & =V_{1}(t)+\frac{1}{2} z_{2}^{2} \\
& =\frac{1}{2} z_{1}^{2}+\frac{1}{2} z_{2}^{2}
\end{aligned}
$$

$$
\dot{V}(t)=z_{1} \dot{z}_{1}+z_{2} \dot{z}_{2}
$$

is negative definite, which means that the solution of the system is asymptotically stable by Theorems 3 and 4 .

The new transformed system is:

$$
{ }_{0}^{C} D_{t}^{\alpha} Y(t)=A Y
$$

where $Y(t)=\left[z_{1}(t) z_{2}(t)+\beta_{1}\left(z_{1}\right)\right]^{T}, \beta=\left[\beta_{1}, \beta_{2}\right]$, and

$$
A=\left[\begin{array}{cc}
-k_{1} & 0 \\
-k_{1} & -2-k_{2}
\end{array}\right], k_{1}, k_{2} \in \mathbb{R}^{+}
$$




\section{Al-Nahrain Journal of Science}

ANJS, Vol.24 (4), December, 2021, pp. 46-51

and it is clear that $A$ has negative eigenvalues $\lambda_{1}=-k_{1}<0$, $\lambda_{2}=-2-k_{2}<0$, for all $k_{1}, k_{2}>0$, which means that the solutions of system (31) are asymptoticly stable.

Sketch of the solutions $y_{1}$ and $y_{2}$ with $k_{1}=1, k_{2}=0.1, \alpha_{1}$ $=0.5$ and $\alpha_{2}=0.7$ are presented in Figure 1 .

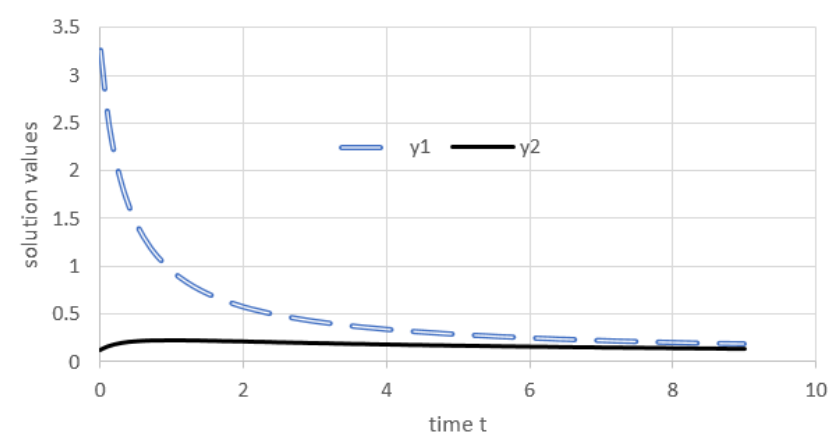

Figure 1. Asymptotic stable solutions of Example 1 with $k_{1}=1, k_{2}=0.1, \alpha_{1}=0.5$ and $\alpha_{2}=0.7$.

Example 2. Consider the linear system of FODEs with Caputo fractional derivative:

$$
\left.\begin{array}{l}
{ }_{0}^{C} D_{t}^{0.65} x(t)=3 x-4 y \\
{ }_{0}^{C} D_{t}^{0.8} y(t)=4 x-7 y+u
\end{array}\right\}
$$

where $x(0) \neq 0, y(0) \neq 0,0<\alpha, \beta \leq 1$.

Step1: Let $z_{1}=x$, and evaluate the Caputo derivative of order 0.65 with respect to $t$, then the first equation of the system Error! Reference source not found. will be as:

$$
{ }_{0}^{C} D_{t}^{0.65} z_{1}(t)=3 z_{1}-4 y
$$

Now, suppose that $z_{2}=y-\beta_{1}$, such that $y=z_{2}+\beta_{1}$, then the equations Error! Reference source not found.), will be:

$$
\begin{aligned}
& { }_{0}^{C} D_{t}^{0.65} z_{1}(t)=3 z_{1}-4 z_{2}-4 \beta_{1} \\
& \text { and } \\
& z_{1}{ }_{0}^{C} D_{t}^{0.65} z_{1}=z_{1}\left(3 z_{1}-4 z_{2}-4 \beta_{1}\right)
\end{aligned}
$$

To do it negative define we choose $\alpha_{1}$ as:

$$
\beta_{1}=\frac{3}{4} z_{1}+\frac{k_{1}}{4} z_{1}
$$

Then:

$$
z_{1}{ }_{0}^{C} D_{t}^{0.65} z_{1}=-k_{1}^{2} z_{1}^{2}-4 z_{1} z_{2}
$$

which is negative everywhere when $k_{1}$ is a positive constant and $z_{2}=0$. The function $\beta_{1}$ should be estimated while $z_{2}$ is considered as a controller.

Since $z_{1}{ }_{0}^{C} D_{t}^{0.65} z_{1}<0$, then by Theorems $2, z_{1} \dot{z_{1}}<0$, that gives $z_{1} \dot{z}_{1}$ will be also negative everywhere and hence if we chose the Lyapunov function $V_{1}=\frac{1}{2} z_{1}^{2}$ and thus $\dot{V}_{1}=$ $z_{1} \dot{z}_{1}$, since $z_{1} \dot{z}_{1}<0$ that mean $\dot{V}_{1}(t)<0$ thus by Theorems 3 and 4 , the solution will be asymptotically stable.

Step2: Let the error between $z_{2}$ and $\beta_{1}$ be:

then

$$
z_{2}=y_{2}-\beta_{1}\left(z_{1}\right)
$$

$$
{ }_{0}^{C} D_{t}^{0.8} z_{2}={ }_{0}^{C} D_{t}^{0.8} y_{2}-{ }_{0}^{C} D_{t}^{0.8} \beta_{1}\left(z_{1}\right)
$$

which will give:

$$
\begin{aligned}
{ }_{0}^{C} D_{t}^{0.8} y_{2} & ={ }_{0}^{C} D_{t}^{0.8} z_{2}+{ }_{0}^{C} D_{t}^{0.8} \beta_{1} \\
& =4 z_{1}-7 z_{2}-7 \beta_{1}\left(z_{1}\right)+u+{ }_{0}^{C} D_{t}^{0.8} \beta_{1}\left(z_{1}\right)
\end{aligned}
$$

and hence:

$$
\begin{gathered}
z_{20}^{C} D_{t}^{0.8} z_{2}=z_{2}\left(4 z_{1}-7 z_{2}-7 \alpha_{1}\left(z_{1}\right)+u+\right. \\
\left.{ }_{0}^{C} D_{t}^{0.8} \alpha_{1}\left(z_{1}\right)\right)
\end{gathered}
$$

to make it negative definite, we choose the controller function as:

$$
u=7 z_{2}-4 z_{1}+7 \alpha_{1}\left(z_{1}\right)-{ }_{0}^{C} D_{t}^{0.8} \alpha_{1}\left(z_{1}\right)-k_{2} z_{2}
$$

and hence:

$$
z_{2}{ }_{0}^{C} D_{t}^{0.8} z_{2}=-k_{2} z_{2}^{2}
$$

which is negative everywhere, and by using by Theorem 2 , since $z_{2}{ }_{0}^{C} D_{t}^{0.8} z_{2}<0$, then $z_{2} \dot{z}_{2}<0$. Tthus, we can choose a Lyapunov function $V_{2}$ as:

$$
V_{2}=V_{1}+\frac{1}{2} z_{2}^{2}=\frac{1}{2} z_{1}^{2}+\frac{1}{2} z_{2}^{2}
$$

such that

$$
\dot{V}_{2}=z_{1} \dot{z}_{1}+z_{2} \dot{z}_{2}
$$

is negative definite and hence the system is asymptotically stable.

Then the equivalence system will be:

$$
{ }_{0}^{C} D_{t}^{\beta} Z(t)=A Z
$$

where $Z(t)=\left[z_{1}(t) z_{2}(t)\right]^{T}, \beta=[0.65,0.8]$ and

$$
A=\left[\begin{array}{cc}
-k_{1} & -4 \\
0 & -k_{2}
\end{array}\right], k_{1}, k_{2} \in \mathbb{R}^{+}
$$

Taking $\mathrm{k} 1=1$ and $\mathrm{k} 2=2$, then:

$$
A=\left[\begin{array}{cc}
-1 & -4 \\
0 & -2
\end{array}\right]
$$

and it is clear that $A$ has negative eigenvalues $\lambda_{1}=-1<0$, $\lambda_{2}=-2<0$, which means that the solutions of system (37) are asymptoticly stable.

Then the solution in terms of Mitag-Liffler form will be:

$$
\begin{aligned}
& z_{1}=E_{\alpha_{1}}\left(-k_{1} t\right)+4 E_{\alpha_{2}}\left(-k_{2} t\right) \\
& z_{2}=E_{\alpha_{2}}\left(-k_{2} t\right)
\end{aligned}
$$

and with respect to $x$ and $y$ is:

$$
\begin{aligned}
& x(t)=E_{\alpha_{1}}\left(-k_{1} t\right)+4 E_{\alpha_{2}}\left(-k_{2} t\right) \\
& y(t)=2 E_{\alpha_{1}}\left(-k_{1} t\right)+4 E_{\alpha_{2}}\left(-k_{2} t\right)
\end{aligned}
$$

Sketch of the solutions $x$ and $y$ are presented in Figure 2 .

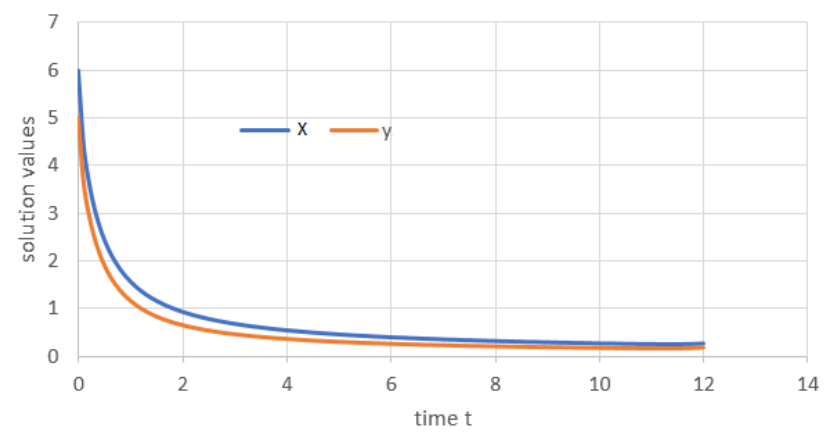

Figure 1. Asymptotic stable solutions of Example.

\section{Conclusion}

Adaptive backstepping method followed in this paper for Caputo FODEs give an easy approach for stabilizing and solving such type of equations by iteratively forming quadratic Lyapunov functions for each subsystem to evaluate one control function which stabilizes the system depending on the theory of fractional order stability theory. The fallowed approach found to be efficient and reliable. 


\section{Al-Nahrain Journal of Science}

ANJS, Vol.24 (4), December, 2021, pp. 46-51

\section{References}

[1] Resnick A.; "Fractional calculus: an introduction for physicists, 3rd edition". Contemp. Phys.; 60(1): 77-77, 2019. doi: $10.1080 / 00107514.2019 .1568582$.

[2] Daftardar-Gejji V. and Babakhani A.; "Analysis of a system of fractional differential equations". J. Math. Anal. Appl.; 293(2): 511-522, 2004. doi: 10.1016/j. jmaa.2004.01.013.

[3] Yang Q.; Chen D.; Zhao T. and Chen Y.; "Fractional calculus in image processing: A review". Fractional Calculus and Applied Analysis, 19(5): 1222-1249, 2016. doi: 10.1515/fca-2016-0063.

[4] Chaid A. R. K. K. M.; "Stability of Linear Multiple Different Order Caputo Fractional System". 6(3): 55-68, 2016.

[5] Petras I.; "Fractional Derivatives, Fractional Integrals, and Fractional Differential Equations in Matlab". Eng. Educ. Res. Using MATLAB, 2011. doi: 10.5772/19412.

[6] Khan A.; Garg N. R. and Jain G.; "Backstepping Design and Fractional Derivative Equation of Chaotic System". Int. J. Math. Comput. Phys. Electr. Comput. Eng, 8(6): 965-969, 2014.

[7] Efe M. O.; "Backstepping control technique for fractional order systems". in The 3rd Conference on Nonlinear Science and Complexity (NSC 2010), (105): $0-5,2010$.

[8] Rozali S. M.; Rahmat M. F.; Husain A. R. and Kamarudin M. N.; "Design of adaptive backstepping with gravitational search algorithm for nonlinear system". J. Theor. Appl. Inf. Technol.; 59(2): 460-468, 2014.

[9] Rudra S.; Barai R. K. and Maitra M.; "Block backstepping design of nonlinear state feedback control law for underactuated mechanical systems". Springer, 2016.

[10] Samko S.; "Fractional integrals and derivatives". November, 1993.

[11] Kilbas A. A.; Srivastava H. M. and J. J. Trujillo, "Today's drugs". Pargyline hydrochloride, 1(5396), 1964.

[12] Herrmann R.; "Fractional calculus: An introduction for physicists". 2011.

[13] Das S.; "Functional Fractional Calculus". 2011.

[14] Hasan N. N. and Hussien D. A.; "Generalized Spline method for integro-differential equations of fractional order". Iraqi J. Sci.; 59(2C): 1093-1099, 2018.

[15] Malinowska A. B. and Torres D. F. M.; "Introduction to the fractional calculus of variations". 2012.

[16] Bolek W.; Sasiadek J. and Wisniewski T.; "Adaptive backstepping control of a power plant station model". in IFAC Proceedings Volumes (IFAC-PapersOnline), 15(1): 215-220, 2002. doi: 10.3182/20020721-6-es1901.01187.

[17] Aguila-Camacho N.; Duarte-Mermoud M. A. and Gallegos J. A.; "Lyapunov functions for fractional order systems". Commun. Nonlinear Sci. Numer. Simul.; 19(9): 2951-2957, $2014 . \quad$ doi: 10.1016/j.cnsns.2014.01.022.
[18] Cong N. D.; Tuan H. T. and Trinh H.; "On asymptotic properties of solutions to fractional differential equations". J. Math. Anal. Appl.; 484(2), 123759, 2020. doi: 10.1016/j.jmaa.2019.123759.

[19] Grimble, M. and Johnson, M.; "Advanced textbooks in control and signal processing". 2013. doi: 10.1007/978-1-4471-4396-3_15.

[20] Koenig H. A.; "Nonlinear Systems". 3. Prentice hall Upper Saddle River, NJ, 2019.

[21] Tian X. and Yang Z.; "Adaptive stabilization of a fractional-order system with unknown disturbance and nonlinear input via a backstepping control technique". Symmetry (Basel).; 12(1), 2020. doi: 10.3390/ SYM12010055.

[22] Rasappan S. and Vaidyanathan S.; "Hybrid synchronization of n-Scroll Chua and Lur'e chaotic systems via backstepping control with novel feedback". Arch. Control Sci.; 22(3): 343-365, 2012. doi: 10.2478/v10170-011-0028-9. 